\title{
Loudness of time-varying stimuli with electric stimulation
}

\author{
Tom Francart ${ }^{\text {a) }}$ \\ ExpORL, Department of Neurosciences, KU Leuven, Herestraat 49 bus 721, B-3000 Leuven, Belgium \\ Hamish Innes-Brown, Hugh J. McDermott, and Colette M. McKay \\ The Bionics Institute, 384-388 Albert Street, East Melbourne, Victoria 3002, Australia
}

(Received 3 October 2013; revised 8 April 2014; accepted 10 April 2014)

\begin{abstract}
McKay, Henshall, Farrell, and McDermott [J. Acoust. Soc. Am. 113, 2054-2063 (2003)] developed a practical method to estimate the loudness of periodic electrical signals presented through a cochlear implant. In the present work, this method was extended to time-varying sounds based on two models of time-varying loudness for normal listeners. To fit the model parameters, loudness balancing data was collected with six cochlear implant listeners. The pulse rate of a modulated pulse train was adjusted to equalize its loudness to a reference stimulus. The stimuli were single-electrode time-limited pulse bursts, repeated at a rate of $50 \mathrm{~Hz}$, with on-times varying between 2 and $20 \mathrm{~ms}$. The parameters of two different models of time-varying loudness were fitted to the results. For each model, parameters defining the time windows over which the electrical pulses contribute to instantaneous loudness were optimized. In each case, a good fit was obtained with the loudness balancing results. Therefore, the practical method was successfully extended to time-varying sounds by combining it with existing models of time-varying loudness for acoustic stimulation.
\end{abstract}

(C) 2014 Acoustical Society of America. [http://dx.doi.org/10.1121/1.4874597]

PACS number(s): 43.66.Cb, 43.66.Mk, 43.66.Ts, 43.66.Ba [EB] Pages: 3513-3519

\section{INTRODUCTION}

When designing stimulation strategies for cochlear implant (CI) stimulation and conducting psychophysical experiments, it is useful to be able to estimate the effect of manipulations of the signal on loudness. To this end, McKay et al. (2003) developed a practical method to estimate the loudness of complex electrical stimuli. The practical method is a simplified version of an earlier model (McKay and McDermott, 1998; McKay et al., 2001), which includes the effect of neural refractoriness but requires many parameters to be estimated for each subject and electrode. This is time consuming and difficult to achieve precisely with current methods. The practical method is based on the assumption that there is on average no net effect of electrode separation and stimulation rate on loudness contributions from each pulse in a pulse train, so total loudness can be estimated as the sum of the loudness contributions of all pulses within a temporal integration window. The method was designed with periodic stimuli in mind, so while it was validated with complex stimuli, the stimuli used were all periodic. This model was not designed to predict the loudness of modulated stimuli in which the modulation period exceeds the duration of the temporal window. In the current study, we extended the practical method to time-varying stimuli. This new model will be useful for the development of new CI stimulation strategies that introduce or enhance slow and deep temporal modulations and therefore influence loudness, with may need to be compensated for (e.g., Laneau et al., 2006; Milczynski et al., 2012; Vandali et al., 2005), strategies that

\footnotetext{
a) Also at: The Bionics Institute, 384-388 Albert Street, East Melbourne VIC 3002, Australia. Author to whom correspondence should be addressed. Electronic mail: tom.francart@med.kuleuven.be
}

normalize loudness (e.g., Francart and McDermott, 2012a,b; McDermott et al., 2003; Varsavsky and McDermott, 2013), or to roughly estimate the effect of temporal modulations on loudness when designing psychophysical and electrophysiological experiments.

For acoustic hearing, there are two phenomenological models that can be used to estimate the loudness of timevarying stimuli. These are the time varying loudness (TVL) model of Glasberg and Moore (2002) and the dynamic loudness model (DLM) of Chalupper and Fastl (2002). Both are based on a model initially proposed by Zwicker (1958).

In the TVL framework, Glasberg and Moore (2002) distinguish between instantaneous loudness, short-term loudness, and long-term loudness. Instantaneous loudness should be interpreted as an intermediary variable which is not available for conscious perception (e.g., neural excitation in the auditory nerve integrated along a temporal window). Shortterm loudness can be described as the loudness perceived at any instant. Long term loudness is the slowly varying loudness that listeners can consciously perceive, or the overall loudness of a relatively short stimulus. Instantaneous loudness is calculated using temporal windows with frequencydependent lengths (Hamming windows of $64 \mathrm{~ms}$ to $2 \mathrm{~ms}$ for frequencies of $20 \mathrm{~Hz}$ to $15000 \mathrm{~Hz}$ ). Short-term loudness is obtained by applying an automatic gain control like circuit to the instantaneous loudness values, with an attack time of $22 \mathrm{~ms}$ and a release time of $50 \mathrm{~ms}$. Long-term loudness is calculated by applying a second automatic gain control like circuit to the short-term loudness values. Glasberg and Moore (2002) validated the TVL model by qualitatively comparing its predictions to data from the literature for the loudness of short sounds of different durations (temporal integration of loudness) and amplitude-modulated sounds. 
We will use the same instantaneous and short/long term loudness terminology throughout this paper.

The DLM (Chalupper and Fastl, 2002) includes an initial temporal analysis window with an equivalent rectangular duration (ERD) of $4 \mathrm{~ms}$, and with respect to the TVL takes another approach to model time-varying loudness by adding a forward-masking stage to the model, which is implemented using an automatic gain control like circuit in each spectral channel with a $5 \mathrm{~ms}$ attack time and a $75 \mathrm{~ms}$ release time. This appends a "tail" to channel-specific loudness values. Temporal integration of instantaneous loudness to short-term loudness is done using a simple first order low-pass filter with $8-\mathrm{Hz}$ cut-off frequency. There is no estimate of longterm loudness. The model was validated by comparing its estimates to data from the literature on temporal integration of loudness and amplitude modulated stimuli.

Rennies et al. (2010) recently compared the TVL and DLM models, using published data on temporal integration of loudness, loudness of sequences of noise bursts, amplitude-modulated sounds, and temporally asymmetric stimuli, all for normal-hearing listeners. They found that both models predicted general trends in the available data well. The DLM model agreed slightly better with loudness data for sounds with strong spectral variations over time, while the TVL model had the advantage of yielding an estimate of long-term loudness. To obtain stimulus loudness values from the models, they used the mean long-term loudness for the TVL model and the peak value of the short-term loudness for the DLM, the latter based on the observation by Zwicker (1977) that the peak short-term loudness value is the dominant aspect.

Loudness with electrical stimulation is generally not as well understood as with acoustic stimulation. This is at least partly due to a limited understanding of the effects on loudness of electrical stimulation of the auditory nerve, and large inter-subject variability due to varying stages of neural degeneration. The available practical method (McKay et al., 2003 ) is therefore less complex than state of the art loudness models for acoustic stimulation. The current proposed extension to the practical method is similarly simple, and it is based on a temporal integration window to be applied to pulse-loudness estimates. McKay et al. (2003) used a rectangular temporal integration window of $2 \mathrm{~ms}$, corresponding to the period of the stimuli, such that it did not have influence on the results. McKay and Henshall (2010) showed that the practical method provided valid loudness estimates for short (500 ms) amplitude modulated pulse trains of 1000 and 8000 pps, with modulation frequencies of 250 and $500 \mathrm{~Hz}$, using a single period ( 4 or $2 \mathrm{~ms}$ ) as the temporal integration window. In their more elaborate model, McKay et al. (2001) used an asymmetric exponential temporal integration window with an ERD of $7 \mathrm{~ms}$, but in that case, the window was applied to neural excitation, rather than pulse loudness contributions. McKay et al. (2013) used the same asymmetric temporal integration window applied to neural excitation, plus a longer one to take into account duration and forward masking effects, and modeled total stimulus loudness as the maximum instantaneous loudness (i.e., integration output) over the entire duration of the stimulus. They used a multi-stage model; in a first stage, the effect of current level on neural excitation was modeled, including a model of refractoriness, using typical parameter values from McKay and McDermott (1998). In a next stage, the temporal integration window was applied with parameter values found by Oxenham (2001) for normal-hearing listeners. The entire model was fitted to loudness balancing data obtained by McKay and McDermott (1998) by fitting the refractoriness parameters rather than the parameters of the temporal integration window. The loudness balancing data for a stimulus with $50-\mathrm{Hz}$ repetition rate were well-explained by the model.

Our main aim was to be able to predict the loudness of time-varying stimuli, rather than very brief sounds. Therefore, we opted to use stimuli longer than $300 \mathrm{~ms}$, rather than the very brief ones typically used in temporal integration studies, in which the effect of stimulus duration on loudness is investigated. We first determined the relationship between perceived loudness and pulse rate at different stimulus duty cycles. Second, we used the results to determine the optimal parameters for two different models of time varying loudness.

In the practical method, a loudness growth function (LGF) consisting of a linear and an exponential part is required for each electrode. Measuring a LGF is very timeconsuming, and difficult to do precisely. We therefore avoided having to measure the LGF by using the same current level for all pulses in the stimulus, and instead varied the stimulation rate. Note that our extension still depends on the basic assumptions of the practical method, i.e., the effects of electrode separation and refractoriness on the loudness contribution of each pulse counterbalance each other on average, such that there is no net effect.

\section{METHODS}

\section{A. Apparatus}

Stimuli were generated off-line in MATLAB using custom scripts. They were delivered using a laptop running the APEX program (Francart et al., 2008), connected to an experimental speech processor provided by Cochlear ${ }^{\circledR}$. In this way, we had exact control over the stimulus. The subjects' clinical sound processors were not used.

\section{B. Subjects}

Six subjects were recruited from the clinical population of the Royal Victorian Eye and Ear Hospital in Melbourne. Some relevant details are shown in Table I. They all had acquired profound hearing impairment post-lingually and were implanted with a Cochlear ${ }^{\circledR}$ Nucleus ${ }^{\circledR}$ CI. Permission to conduct the studies was obtained from the Human Research and Ethics Committee of the Royal Victorian Eye and Ear Hospital, and each participant provided written informed consent. Subjects participated in up to four test sessions of 1 to $2 \mathrm{~h}$ duration.

\section{Stimuli}

The reference stimulus was a single-electrode time-limited pulse burst, repeated at a rate of $50 \mathrm{~Hz}$, with an on-time 
TABLE I. Subject info. Age and duration of CI use are expressed in years at the time of testing.

\begin{tabular}{|c|c|c|c|c|c|c|}
\hline Subject & Age (y) & CI use (y) & Implant type & CI side & Aetiology & Electrode \\
\hline N030 & 62 & 16 & Freedom Implant (Contour advance) & $\mathrm{R}$ & Meningitis & 13 \\
\hline N031 & 79 & 11 & CI24M - Nucleus 24 & $\mathrm{R}$ & Hereditary/unknown & 13 \\
\hline N141 & 74 & 4 & Freedom Implant (Contour advance) & $\mathrm{R}$ & Unknown/genetic - post-lingual & 13 \\
\hline N154 & 39 & 10 & CI24R (CS) - Nucleus 24 Contour & $\mathrm{R}$ & Hereditary/unknown & 5 \\
\hline N155 & 58 & 7 & CI24R (CA) - Nucleus 24 Contour Advance & $\mathrm{R}$ & Viral - rubella & 13 \\
\hline N156 & 79 & 4 & Freedom implant (contour advance) & $\mathrm{R}$ & Hereditary/unknown & 13 \\
\hline N157 & 80 & 8 & $\mathrm{CI} 24 \mathrm{R}(\mathrm{CS})$ & $\mathrm{R}$ & Meniere's & 13 \\
\hline
\end{tabular}

of $2 \mathrm{~ms}$ (duty cycle 0.1 ) and a pulse rate of either $7200 \mathrm{pps}$ or 14200 pps. The entire stimulus was $400 \mathrm{~ms}$ long. All pulses were presented at the same current level, determined during an initial part of the procedure (see below). By using same current level we avoided having to measure a full LGF to be able to do the analysis. In Fig. 1, part of the stimulus is illustrated. For the test stimulus the on-time varied from 3 to $20 \mathrm{~ms}$ (duty cycle 0.15 to 1 ). The pulse rate of the test stimulus was determined by an adaptive procedure (see below).

All stimuli were presented on the same electrode for each participant, which was selected in the middle of the array (electrode 13 of 22 for most participants), and of which the clinical threshold $(\mathrm{T})$ and comfortable $(\mathrm{C})$ levels did not deviate much from those of the neighboring electrodes. The electrode used for each subject is shown in Table I. All pulses were presented in monopolar mode $(\mathrm{MP} 1+2)$, with a phase width of 25 us and an inter-phase gap of 8 us.

\section{Procedure}

Loudness balancing experiments in which the pulse rate of a test stimulus was adjusted such that it sounded equally loud as the reference stimulus were performed. There were two reference stimuli. In conditions R7200 and R14200, the pulse rate of the reference stimulus was set at $7200 \mathrm{pps}$ or $14200 \mathrm{pps}$, respectively. The on-time of the reference

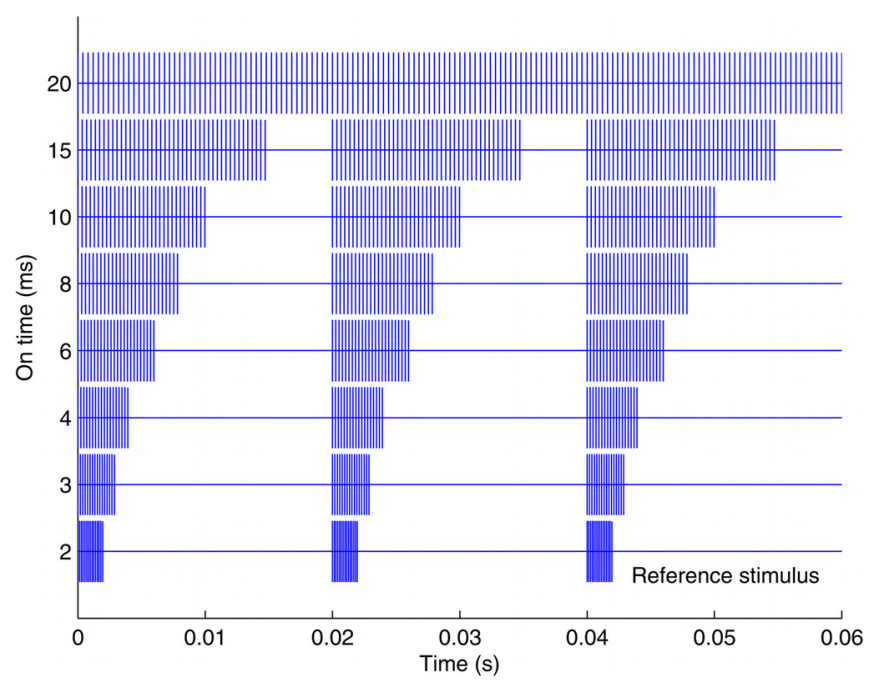

FIG. 1. (Color online) Fragment of the stimulus for the various on-times used in condition R14200, with a rate of $7200 \mathrm{pps}$ for the reference stimulus and rates that yielded an approximately equal loudness percept for the remaining on-times. The stimulus with on-time $2 \mathrm{~ms}$ was used as a reference. Vertical lines indicate biphasic pulses. stimulus was $2 \mathrm{~ms}$. Its current level was set such that it sounded comfortably loud. In each condition, a number of test on-times were used. For R7200, the test on-times were 4 and $10 \mathrm{~ms},{ }^{1}$ and for R14200 the test on-times were 3, 4, 6, 8, 10, 15 , and $20 \mathrm{~ms}$.

The balancing procedure was an adaptive 2-interval, 2alternative forced-choice (2I-2AFC) 1up-1down procedure, adjusting the pulse rate of the test stimulus. The initial step size was a $20 \%$ pulse rate change. After four reversals it was reduced to $10 \%$. A run was stopped after 14 reversals. The subject's task was to indicate which interval was louder, the first or second. The test and reference stimuli were presented in random order. The loudness balanced pulse rate was calculated as the geometric mean of the last six reversals. For each test on-time value, at least two runs were conducted, and the geometric average was calculated.

The high reference rates were chosen to avoid the subjects perceiving a rate pitch at lower matched rates and to maintain the freedom to manipulate the pulse rate (and corresponding number of pulses per period) for short on-times.

\section{RESULTS}

The results of the balancing experiments are shown in Fig. 2 and in Table II for future reference. Individual results and the grand average are shown. The dashed line indicates the balance that could be expected based purely on the total number of pulses in each stimulus, assuming that an equal number of pulses would lead to equal loudness. Additionally, in condition R7200, the average for R14200 is shown, but it is transposed to R7200 by multiplying all points by $7200 / 14200$.

The results show that as the on-time increases from the reference of $2 \mathrm{~ms}$, a lower pulse rate is needed to maintain the same perception of loudness. However, the total number of pulses required for equal loudness increases with increasing on-time. This can be seen by considering the difference between the dashed line and the measurement results in Fig. 2. This was the case for reference rates of 7200 and 14200 pps, although the effect was slightly stronger in the 7200 pps condition. It means that short on-times yield a louder percept for the same number of pulses. To test the statistical significance of these effects, the loudness balanced rates were submitted to a repeated measures analysis of variance (ANOVA) with factors on-time (4 or $10 \mathrm{~ms}$ ) and reference rate $(7200 \mathrm{pps}$ or $14200 \mathrm{pps}$ transposed to 7200 pps). The ANOVA revealed a significant effect of on-time $[F(1,4)=74.3, p<0.01]$ and a significant effect of reference 

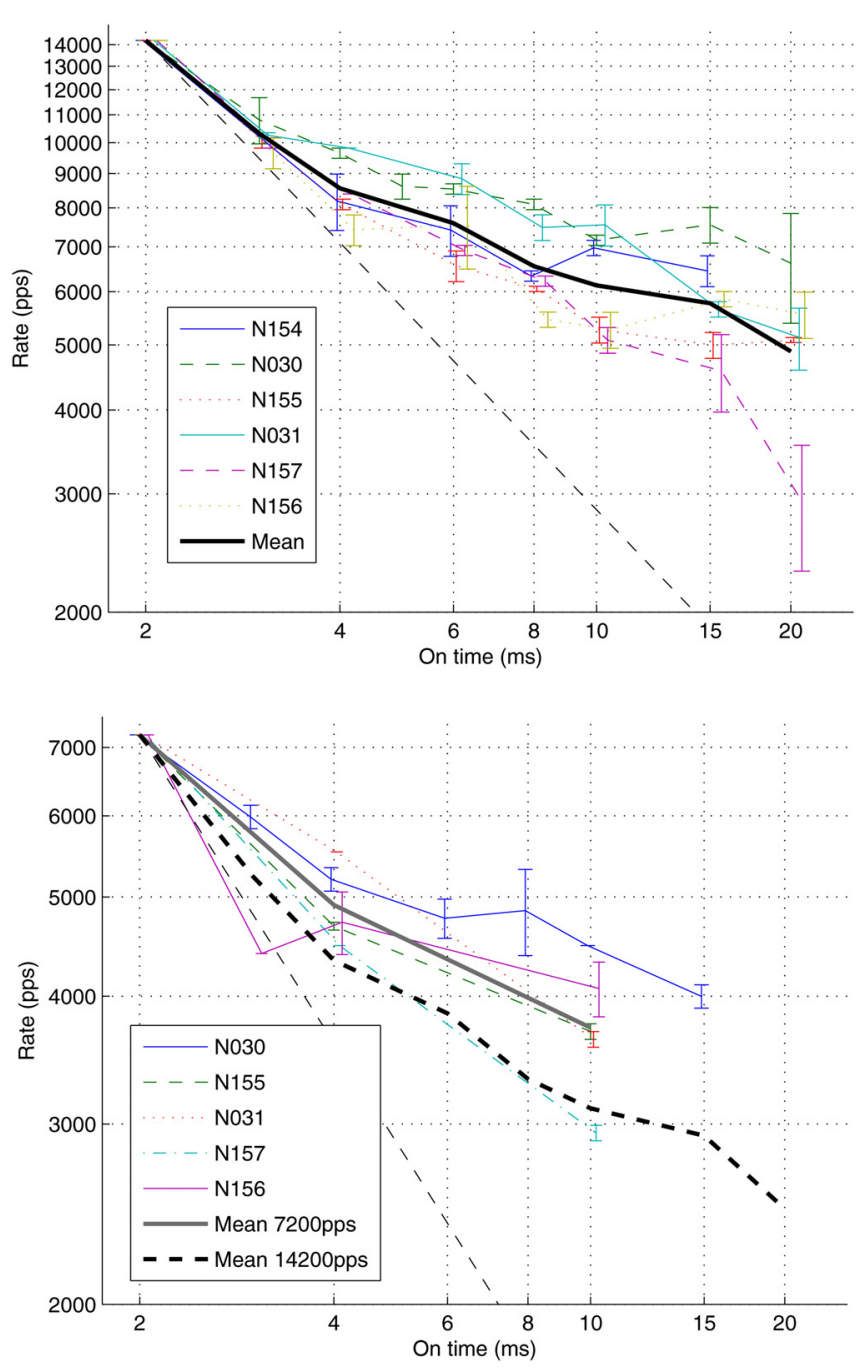

FIG. 2. (Color online) Balancing results. Each point indicates the rate required to make the stimulus with on-time indicated on the $\mathrm{X}$-axis equally loud as the stimulus with on-time $2 \mathrm{~ms}$, averaged across several runs. The error bars indicate the corresponding standard deviations. The top plot shows the results for a reference rate of $14200 \mathrm{pps}$ and the bottom plot for 7200 pps. The thick black line in the first panel and thick gray line in the second panel show the geometric mean value across subjects. The dashed black line in the bottom plot indicates the across subject mean for the 14200 pps reference, but transposed to 7200 pps. The straight dashed line in both panels indicates the rate that would be obtained if the pulses in the entire stimulus contributed equally to loudness.

rate $[F(1,4)=11.4, p=0.03]$. Balanced rates were slightly lower for R14200. It should be noted that when comparing between the $2 \mathrm{~ms}$ and $20 \mathrm{~ms}$ stimuli, most subjects perceived a significant pitch difference, which might have influenced

TABLE II. Balanced rates in pps for different on-times, for a reference carrier rate of $14200 \mathrm{pps}$ and on-time of $2 \mathrm{~ms}$.

\begin{tabular}{lcccccccc}
\hline \hline Subject & $2 \mathrm{~ms}$ & $3 \mathrm{~ms}$ & $4 \mathrm{~ms}$ & $6 \mathrm{~ms}$ & $8 \mathrm{~ms}$ & $10 \mathrm{~ms}$ & $15 \mathrm{~ms}$ & $20 \mathrm{~ms}$ \\
\hline $\mathrm{N} 154$ & 14200 & 11195 & 8191 & 7415 & 6331 & 6973 & 6443 & - \\
N030 & 14200 & 10816 & 9650 & 8533 & 8096 & 7159 & 7546 & 6615 \\
N155 & 14200 & 10083 & 8096 & 6558 & 6059 & 5265 & 4995 & 5083 \\
N031 & 14200 & 10262 & 9821 & 8839 & 7480 & 7546 & 5648 & 5128 \\
N157 & 14200 & 9995 & 8385 & 6912 & 6221 & 5083 & 4575 & 2923 \\
N156 & 14200 & 9650 & 7415 & 7546 & 5453 & 5265 & 5850 & 5550 \\
\hline \hline
\end{tabular}

TABLE III. $R^{2}$ values for a selection of conditions (including or excluding the balanced rate for on-time $20 \mathrm{~ms}$ ), scales (linear on-time versus linear rate or logarithm of on-time versus logarithm of rate), and functions (linear or power). In condition No20 the data point for on-time $=20 \mathrm{~ms}$ was excluded from the fit.

\begin{tabular}{lccc}
\hline \hline Condition & Scale & Function & $R^{2}$ \\
\hline All & log-log & power & 0.992 \\
All & $\log -\log$ & linear & 0.955 \\
All & lin-lin & power & 0.953 \\
All & $\operatorname{lin}-\operatorname{lin}$ & linear & 0.641 \\
No20 & $\log -\log$ & power & 0.995 \\
No20 & $\log -\log$ & linear & 0.943 \\
No20 & lin-lin & power & 0.950 \\
No20 & lin-lin & linear & 0.652 \\
\hline \hline
\end{tabular}

the results. For the other comparisons, no pitch differences were reported.

We attempted to fit a large number of functions to the on-time versus rate data. An overview of $R^{2}$ values for a selection of scales (linear on-time versus linear rate, or logarithm of on-time versus logarithm of rate) and functions (linear or power) is shown in Table III. We conducted the fit with and without inclusion of the pulse rate for on-time $20 \mathrm{~ms}$, as this point might be biased due to an associated difference in pitch percept. The best fit for a linear and power function is shown in Fig. 3. The best fit was obtained by fitting a power function on a logarithmic on-time versus logarithmic rate scale, excluding on-time $20 \mathrm{~ms}$, according to $\log ($ rate $)=3.80[\log (\text { on-time })]^{-0.074}$.

\section{MODELING}

We determined the optimum temporal integration window duration for the TVL and an alternative model to be used in conjunction with the practical method to estimate the loudness of complex electrical stimuli (McKay et al., 2003). Overall, in our balancing results, there was some variability

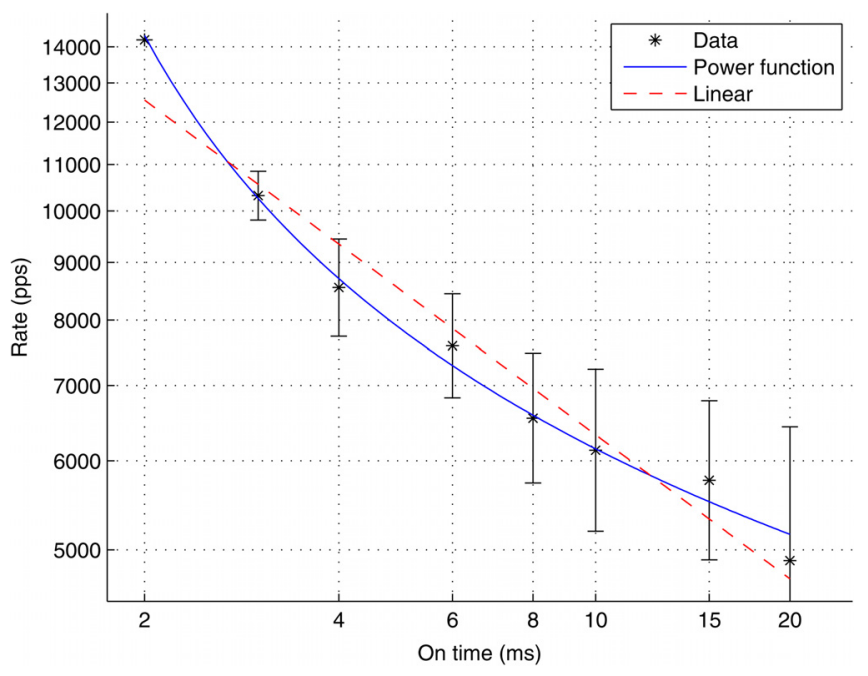

FIG. 3. (Color online) Mean on-time versus rate data on a log-log scale, fitted by a power function and linear function. The error bars indicate acrosssubject geometric standard deviations. 
across subjects, but all curves showed the same tendency. Therefore, for the modeling work, the average was used.

In the literature, asymmetric exponential temporal integration windows are most often used. Plack and Oxenham (1998) defined a temporal window to integrate basilar membrane response as follows:

$$
\left\{\begin{array}{lrl}
W(t) & =(1-w) e^{t / T_{b 1}}+w e^{t / T_{b 2}}, & t<0, \\
W(t)=e^{t / T_{a}}, & t>0
\end{array}\right.
$$

with $T_{a}=3.5, T_{b 1}=4.6, T_{b 2}=16.6$, and $w=0.17$.

McKay and McDermott (1998) integrated neural excitation using an asymmetric exponential time window $W(t)$ with time constants on each side $\tau_{1}$ and $\tau_{1}$ such that $\tau_{1}=0.63 \tau_{2}$

$$
\left\{\begin{array}{l}
W(t)=e^{t / \tau_{1}}, \quad t<0, \\
W(t)=e^{-t / \tau_{2}}, \quad t>0
\end{array}\right.
$$

with $t$ time in ms. This is a special case of the Plack and Oxenham (1998) window. The ERD, defined as $\tau_{1}+\tau_{2}$, was set to $7 \mathrm{~ms}$, based on parameters found by Moore et al. (1996).

In Fig. 4, we plotted instantaneous loudness as a function of time for each stimulus (only $100 \mathrm{~ms}$ of the stimulus is shown in the figure), for the loudness balanced rates obtained from our experiments, using the McKay and McDermott (1998) window for $\mathrm{ERD}=3,5$, or $7 \mathrm{~ms}$. Note that we used an arbitrary pulse loudness contribution of 1 for each pulse, so all results should be considered on a relative scale. For a very short ERD (3 ms) the stimuli with shorter on-times had higher instantaneous-loudness peak values because their rate was higher. As the stimuli with different on-times were balanced in loudness, the model should predict equal long-term loudness. For the $3 \mathrm{~ms}$ ERD, reasonable results could be obtained using the TVL, in which the attack/release time approach smooths (lowers) the narrow peaks for short ontimes, yielding similar total loudness values for all stimuli. For longer ERDs (5 and $7 \mathrm{~ms}$ ), the maximum instantaneous

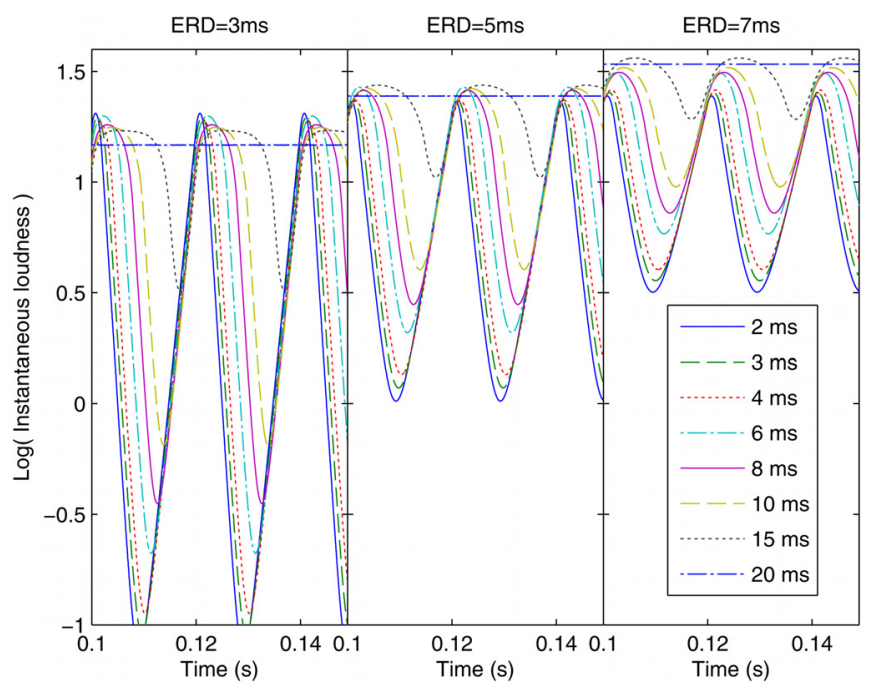

FIG. 4. (Color online) Instantaneous loudness calculated using the McKay and McDermott (1998) window for $\mathrm{ERD}=3,5$, or $7 \mathrm{~ms}$, for the pulse rates obtained from the loudness balancing experiments. Different colors and line styles indicate the different on-times shown in the legend. loudness was lower for shorter on-times and neither the attack/release time approach of the TVL, nor low-pass filtering and taking the maximum instantaneous loudness (DLM) can predict a long-term loudness that is higher than the maximal instantaneous loudness of the narrow peaks obtained for short on-times. It seems therefore unlikely that ERDs longer than $5 \mathrm{~ms}$ would yield a good fit.

We calculated the short-term loudness using the TVL with an ERD of $5 \mathrm{~ms}$ applied to our data. The results are shown in Fig. 5. It is clear that whatever method is used to obtain long-term loudness, it will never be the similar for all on-times for this ERD. We also ran an optimization routine to find the attack time, release time, and ERD of the integration window that yielded the smallest standard deviation of long-term loudness across on-times. When we allowed the algorithm to select very short ERDs $(2-3 \mathrm{~ms})$, these were preferred together with very short attack times (0-1 ms) and release times of $20-40 \mathrm{~ms}$. While the release time was in the order of the $50 \mathrm{~ms}$ that Glasberg and Moore (2002) used, and the ERD of 2-3 ms was similar to what they used for high frequencies, the attack time was much shorter. The lowest standard deviation of the logarithm of total loudness was 0.0153 for $\mathrm{ERD}=2 \mathrm{~ms}$, attack time $1 \mathrm{~ms}$ and release time $20 \mathrm{~ms}$. If the algorithm was constrained to ERDs above $4 \mathrm{~ms}$ to conform to McKay and McDermott (1998), 0-ms attack times and very long release times were obtained.

In a second approach, we considered a simpler model: Instantaneous loudness was calculated as before, and longterm loudness was calculated as the $N$ th percentile instantaneous loudness value across the stimulus. This was inspired by the approach taken by Rennies et al. (2010), who used the peak output of the DLM. We used a percentile value instead, because it seems physiologically unlikely that total loudness would be determined by a single instantaneous loudness value. We again calculated the standard deviation across ontimes. The lowest standard deviations were found for high percentile values (99\%), and for an ERD of $4.3 \mathrm{~ms}$. The standard deviation in the best condition was 0.0215 , slightly higher than for the Glasberg and Moore (2002) method. We
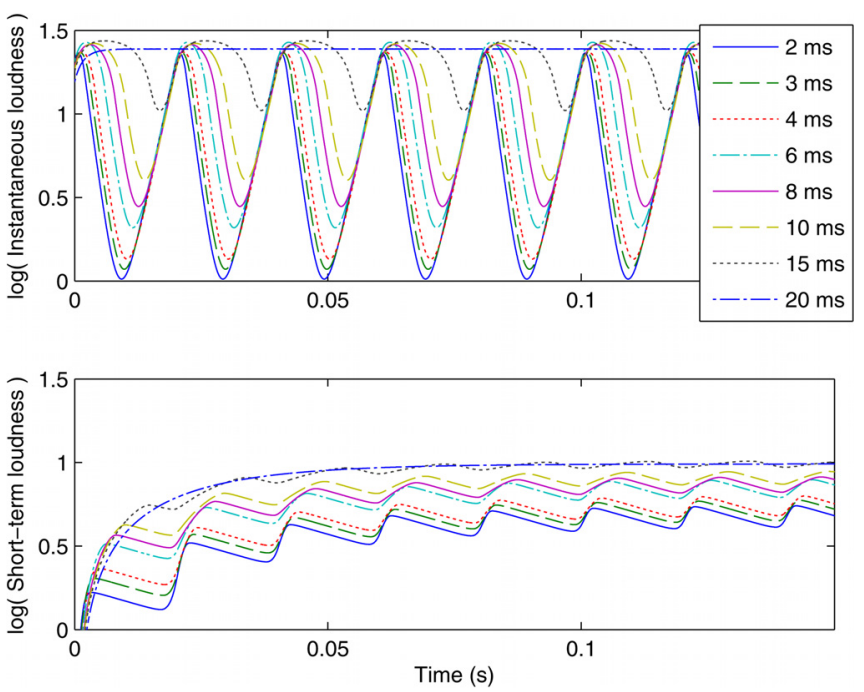

FIG. 5. (Color online) Short term loudness calculated according to Glasberg and Moore (2002) for ERD $=5 \mathrm{~ms}$. 
also investigated the use of the window proposed by Plack and Oxenham (1998) and a rectangular window, but neither yielded better results.

The $20 \mathrm{~ms}$ on-time stimulus yielded a different percept, so the balancing result might be biased. We therefore repeated the analysis above excluding this data point. The obtained model parameters remained similar $(E R D=2 \mathrm{~ms}$, attack time $2 \mathrm{~ms}$, and release time $40 \mathrm{~ms}$ for the TVL, and $\mathrm{ERD}=4.2 \mathrm{~ms}$ for the percentile method), but the corresponding standard deviations across on-times decreased to around 0.0119 for the TVL method and around 0.0159 for the percentile method. The subjects did not report any pitch differences for on-times shorter than $20 \mathrm{~ms}$. Should there have been an unreported pitch difference anyway, it could have influenced the balanced rate estimates in a similar way, leading to a similar increase in standard deviation.

Finally, we used balanced rate values obtained from the fit of a power function on a log-log scale (cf. Fig. 3). This yielded similar model parameters $(E R D=2 \mathrm{~ms}$, attack time $2 \mathrm{~ms}$, and release time $30 \mathrm{~ms}$ for the TVL, and ERD $=4.3 \mathrm{~ms}$ for the percentile method), but lower standard deviations across on-time: 0.0079 and 0.0121 for the TVL and percentile method, respectively.

When using the TVL or percentile model with optimal parameters to predict balanced rates, we obtained the results shown in Fig. 6. While the differences are minor, the fit for the TVL appears slightly better.

\section{DISCUSSION}

The practical method to estimate the loudness of electrical stimuli developed by McKay et al. (2003) was extended for time-varying stimuli by combining it with the TVL model and an alternative model. We determined the appropriate ERD of the temporal integration window using loudness balancing data between $50-\mathrm{Hz}$ modulated pulse trains with different on-times.

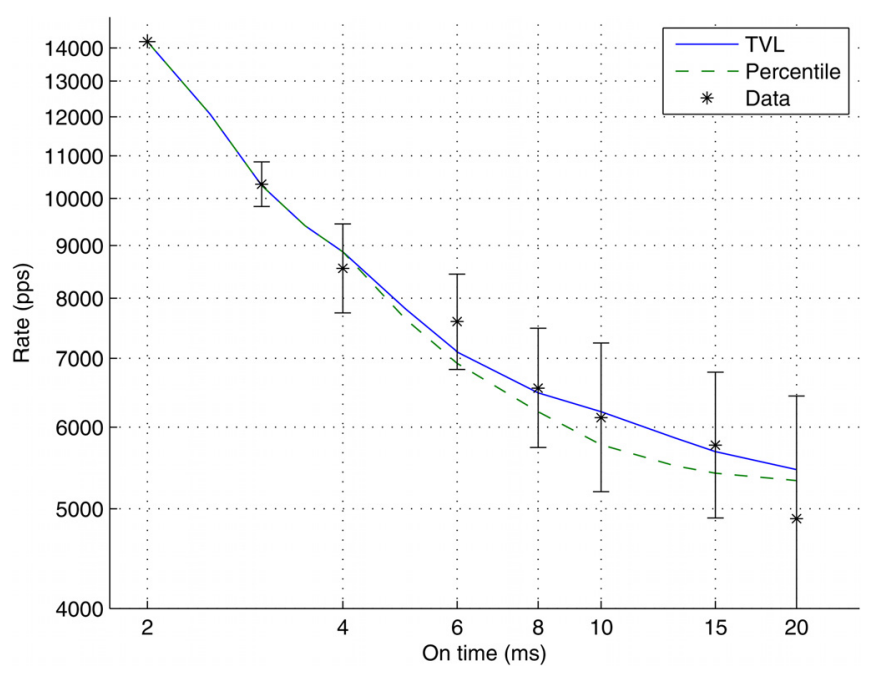

FIG. 6. (Color online) Balanced rates measured versus predicted by the two models. For the TVL model (Glasberg and Moore, 2002) the parameters were $\mathrm{ERD}=2 \mathrm{~ms}$, attack time $1 \mathrm{~ms}$, and release time $40 \mathrm{~ms}$. For the percentile model the 99th percentile and an ERD of $4.2 \mathrm{~ms}$ were used. The error bars indicate across-subject geometric standard deviations.
Comparing the balancing results in conditions R14200 (transposed to $7200 \mathrm{pps)}$ and R7200, it was found that balanced rates were slightly lower for R14200. While this might seem an effect of refractoriness not predicted by the practical method (McKay et al., 2003), this is probably not the case because one would expect more refractoriness at higher rates, and thus higher pulse rates would be required to match the reference. It should be pointed out though that for R7200 only a few data points were measured, and there is quite some variability across subjects, so this finding should be interpreted with caution. Our main aim was to extend the practical method, which is only approximately correct for average data, and only models refractory effects to the extent that they are balanced against other factors, unlike the more extensive loudness model developed by McKay and McDermott (1998), McKay et al. (2001), and McKay et al. (2013).

When comparing temporal integration window ERDs across studies, one should always take into account that (1) the ERD depends on the physical unit being integrated, for instance, when signal intensity versus signal intensity after non-linear compression are integrated, different ERD values will be found for the same process (McKay and McDermott, 1998; Oxenham and Moore, 1994; Penner, 1978), and (2) the ERD is usually part of a larger model, of which multiple parameters are fitted together. Often the parameters are not independent, which means that the obtained ERD values are only meaningful in conjunction with the rest of the model.

McKay and McDermott (1998) used a sliding temporal integration window, applied to neural excitation. They attempted to fit the ERD together with the three other parameters in their model. For five out of eight subjects, this was unsuccessful because the other parameters depended too much on ERD, and for the remaining three subjects they found values of 7.5, 5.3, and $6.8 \mathrm{~ms}$. Based on earlier work by Moore et al. (1996), they decided to use an ERD of $7 \mathrm{~ms}$ for all subjects except one, for whom a successful model fit could only be obtained for ERDs smaller than $5.5 \mathrm{~ms}$. Given that in the current study we have integrated another measure (pulse loudness contribution) and that the obtained ERD of $4.3 \mathrm{~ms}$ is not much lower than the values found by McKay and McDermott (1998), there is no reason to assume that their model would be in disagreement with the current one.

For the percentile method, we obtained an ERD of $4.2 \mathrm{~ms}$, which is well in accordance with the $4 \mathrm{~ms}$ used in the DLM. For the TVL, we obtained optimal parameter values of ERD $=2 \mathrm{~ms}$, attack time $2 \mathrm{~ms}$, and release time $20-40 \mathrm{~ms}$, which except for the attack time is in accordance with the published parameters of ERD $=2-64 \mathrm{~ms}$, attack time $22 \mathrm{~ms}$ and release time $50 \mathrm{~ms}$ (Glasberg and Moore, 2002). The similarities between the current results obtained with electric stimulation and the literature on acoustic stimulation suggest that the mechanisms behind the integration of instantaneous loudness into short or long term loudness are similar for both stimulation modes.

In conclusion, we found that the practical loudness estimation method for electrical stimulation developed by McKay et al. (2003) can be extended to time-varying stimuli by using a temporal integration window with an ERD of 
around $2 \mathrm{~ms}$ when subsequently calculating long-term loudness according to Glasberg and Moore (2002), or an ERD of around $4.3 \mathrm{~ms}$ when estimating the total loudness as the 99th percentile of the instantaneous loudness. We expect that this extension of the model will be useful for the development of new CI stimulation strategies that influence the temporal characteristics of the signal and therefore the loudness, and to roughly estimate the effect of temporal modulations on loudness when designing psychophysical experiments.

\section{ACKNOWLEDGMENTS}

We are grateful to our test subjects, who voluntarily participated in numerous test sessions. T.F. was sponsored by a Post Doctoral Fellowship of the Fund for Scientific Research of the Flemish Government, a Marie Curie International Outgoing Fellowship of the European Commission, grant agreement number PIOF-GA-2009-474 252730, and IWTVlaanderen project 110722. The Bionics Institute acknowledges the support it receives from the Victorian Government through its Operational Infrastructure Support Program.

${ }^{1}$ In pilot experiments with two subjects other on-times were tested in condition R7200. These data points are shown in the figures, but they were not further taken into account in the analysis.

Chalupper, J., and Fastl, H. (2002). "Dynamic loudness model (DLM) for normal and hearing-impaired listeners," Acta Acust. Acust. 88, 378-386.

Francart, T., and McDermott, H. J. (2012a). "Development of a loudness normalisation strategy for combined cochlear implant and acoustic stimulation," Hear. Res. 294, 114-124.

Francart, T., and McDermott, H. J. (2012b). "Speech perception and localisation with SCORE bimodal: A loudness normalisation strategy for combined cochlear implant and hearing aid stimulation," PLoS One 7, e45385.

Francart, T., van Wieringen, A., and Wouters, J. (2008). "APEX 3: A multipurpose test platform for auditory psychophysical experiments," J. Neurosci. Methods 172, 283-293.

Glasberg, B. R., and Moore, B. (2002). "A model of loudness applicable to time-varying sounds,” J. Audio Eng. Soc. 50, 331-342.

Laneau, J., Wouters, J., and Moonen, M. (2006). "Improved music perception with explicit pitch coding in cochlear implants," Audiol. Neurootol. 11, 38-52.
McDermott, H., McKay, C. M., Richardson, L. M., and Henshall, K. R. (2003). "Application of loudness models to sound processing for cochlear implants," J. Acoust. Soc. Am. 114, 2190-2197.

McKay, C. M., and Henshall, K. R. (2010). "Amplitude modulation and loudness in cochlear implantees," J. Assoc. Res. Otolaryngol. 11, 101-111.

McKay, C. M., Henshall, K. R., Farrell, R. J., and McDermott, H. (2003). "A practical method of predicting the loudness of complex electrical stimuli,” J. Acoust. Soc. Am. 113, 2054-2063.

McKay, C. M., Lim, H. H., and Lenarz, T. (2013). "Temporal processing in the auditory system: Insights from cochlear and auditory midbrain implantees,” J. Assoc. Res. Otolaryngol. 14, 103-124.

McKay, C. M., and McDermott, H. (1998). "Loudness perception with pulsatile electrical stimulation: The effect of interpulse intervals," J. Acoust. Soc. Am. 104, 1061-1074.

McKay, C. M., Remine, M. D., and McDermott, H. (2001). "Loudness summation for pulsatile electrical stimulation of the cochlea: Effects of rate, electrode separation, level, and mode of stimulation," J. Acoust. Soc. Am. 110, 1514-1524.

Milczynski, M., Chang, J. E., Wouters, J., and van Wieringen, A. (2012). "Perception of Mandarin Chinese with cochlear implants using enhanced temporal pitch cues," Hear. Res. 285, 1-12.

Moore, B. C., Peters, R. W., and Glasberg, B. R. (1996). "Detection of decrements and increments in sinusoids at high overall levels," J. Acoust. Soc. Am. 99, 3669-3677.

Oxenham, A. J. (2001). "Forward masking: Adaptation or integration?" J. Acoust. Soc. Am. 109, 732-741.

Oxenham, A. J., and Moore, B. C. (1994). "Modeling the additivity of nonsimultaneous masking," Hear. Res. 80, 105-118.

Penner, M. J. (1978). "A power law transformation resulting in a class of short-term integrators that produce time-intensity trades for noise bursts," J. Acoust. Soc. Am. 63, 195-201.

Plack, C. J. and Oxenham, A. J. (1998). "Basilar-membrane nonlinearity and the growth of forward masking," J. Acoust. Soc. Am. 103, 1598-1608.

Rennies, J., Verhey, J. L., and Fastl, H. (2010). "Comparison of loudness models for time-varying sounds," Acta Acust. Acust. 96, 383-396.

Vandali, A. E., Sucher, C. M., Tsang, D. J., McKay, C. M., Chew, J. W., and McDermott, H. (2005). "Pitch ranking ability of cochlear implant recipients: a comparison of sound-processing strategies," J. Acoust. Soc. Am. 117, 3126-3138.

Varsavsky, A., and McDermott, H. J. (2013). “Application of real-time loudness models can improve speech recognition for cochlear implant users," IEEE Trans. Neural Syst. Rehabil. Eng. 21, 81-87.

Zwicker, E. (1958). "Ueber psychologische und methodische Grundlagen der Lautheit" ("On psychological and methodical principles of loudness"), Acustica 8, Suppl. 1, 237-258.

Zwicker, E. (1977). "Procedure for calculating loudnesss of temporally variable sounds," J. Acoust. Soc. Am. 62, 675-682. 